\title{
Satellite Imaging of Global Urbanicity relate to Adolescent Brain Development and Behavior
}

Jiayuan $\mathrm{Xu}^{1,2}$, Xiaoxuan $\mathrm{Liu}^{3,4}$, Alex Ing ${ }^{2}$, Qiaojun $\mathrm{Li}^{5,2}$, Wen Qin ${ }^{1}$, Le Yu ${ }^{3,4}$, Chunshui Yu ${ }^{1 *}$, Gunter Schumann ${ }^{2 *}$, and the CHIMGEN and IMAGEN Consortia

1. PONS center, SGDP, Institute of Psychiatry, Psychology and Neuroscience, King 's College London, London SE5 8AF, United Kingdom

2. Department of Radiology and Tianjin Key Laboratory of Functional Imaging, Tianjin Medical University General Hospital, Tianjin P.R. China

3. Ministry of Education Key Laboratory for Earth System Modeling, Department of Earth System Science, Tsinghua University, Beijing 100084, P.R. China

4. Joint Center for Global Change Studies, Beijing 100875, P.R. China

5. College of Information Engineering, Tianjin University of Commerce, Tianjin P.R. China

Poster \# 17

\section{Aim}

- Using innovative remote-sensing satellite data acquisition, to investigate whether structural and functional brain differences and behaviours related to urbanicity are similar or distinct in Chinese and European participants

$\checkmark$ To investigate possible susceptibility windows for the effects of urbanicity during child and adolescent development

\section{Background}

- Urbanicity, the impact of living in urban areas, is among the greatest environmental challenges for mental health. While urbanicity might be distinct in different sociocultural conditions and geographic locations, there are likely to exist common features shared in different areas of the globe

Understanding these common and specific relations of urbanicity with human brain and behaviour will enable to assess the impact of urbanicity on mental disorders, especially in childhood and adolescence, where prevention and early interventions are likely to be most effective

- Satellite-based remote sensing data was used to construct a factor for urbanicity-'UrbanSat', which was highly correlated with population density ground data

\section{Datasets}

- Chinese CHIMGEN sample (N=831)

- longitudinal European IMAGEN cohort $(\mathrm{N}=810)$

,

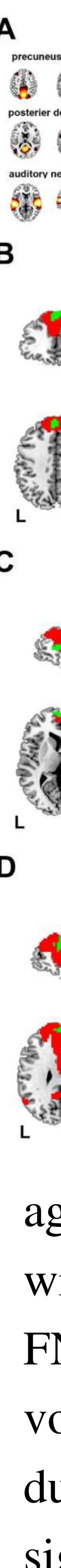

Figure 3. Voxel wise correlations

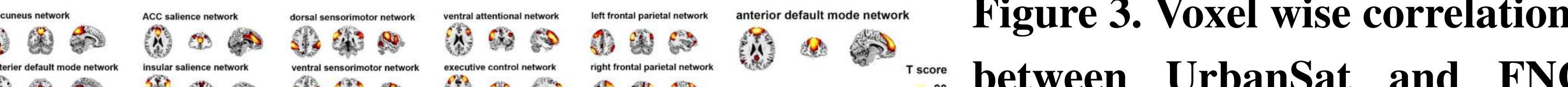
independent resting state

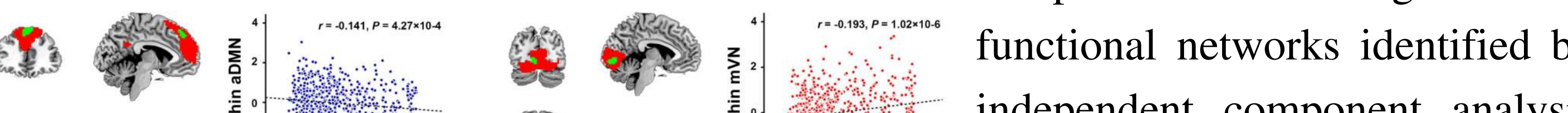
T) $=$ mPFC

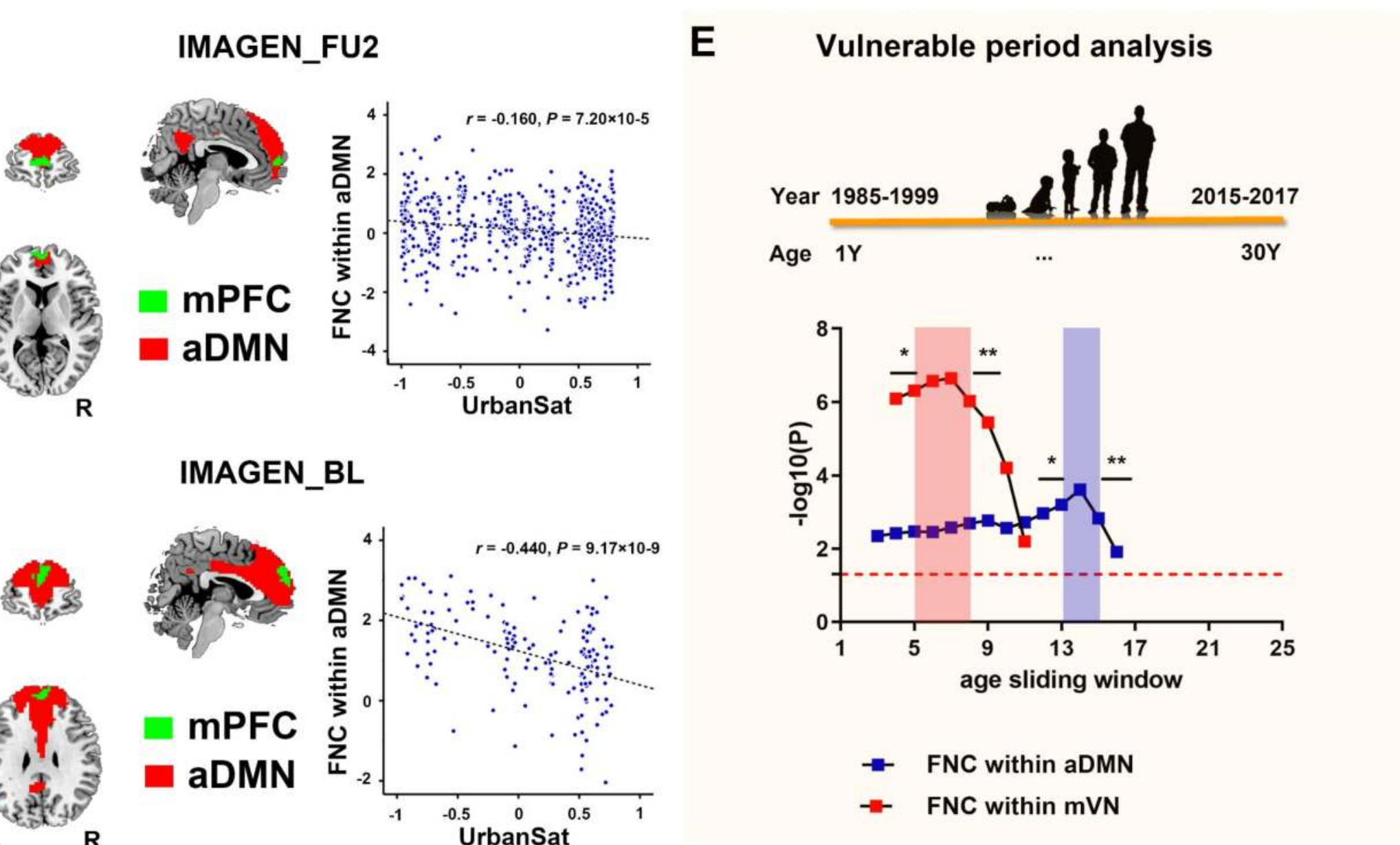
the CHIMGEN, a significant negative correlation of UrbanSa before age 18 years with FNC within the left $\mathrm{mFPC}$ of the aDMN (left) and a significant positive correlation with FNC within the left $\mathrm{LG}$ of the $\mathrm{mVN}$ (right) (FWE-correction, voxe P<0.05); C. In the IMAGEN at

age 19 years, a significant negative correlation of UrbanSat at 19 years with FNC within the aDMN (FWE-correction, voxel $\mathrm{P}<0.05$ ). D. The correlation of UrbanSat on FNC within the aDMN was also present in the IMAGEN at 14 years (FWE-correction, voxel P<0.05). E. The correlation of UrbanSat with FNC within the aDMN was highest during adolescence (ages 13 to 15 years). The difference to the adjacent age bands was significant compared to age 12 years $(\mathrm{P}=0.010)$ and to age 16 years $(\mathrm{P}=6.16 \times 10-5)$; The correlation of UrbanSat with $\mathrm{FNC}$ within the $\mathrm{mVN}$ was only vulnerable childhood and highest during childhood (ages 5 to 8 years).

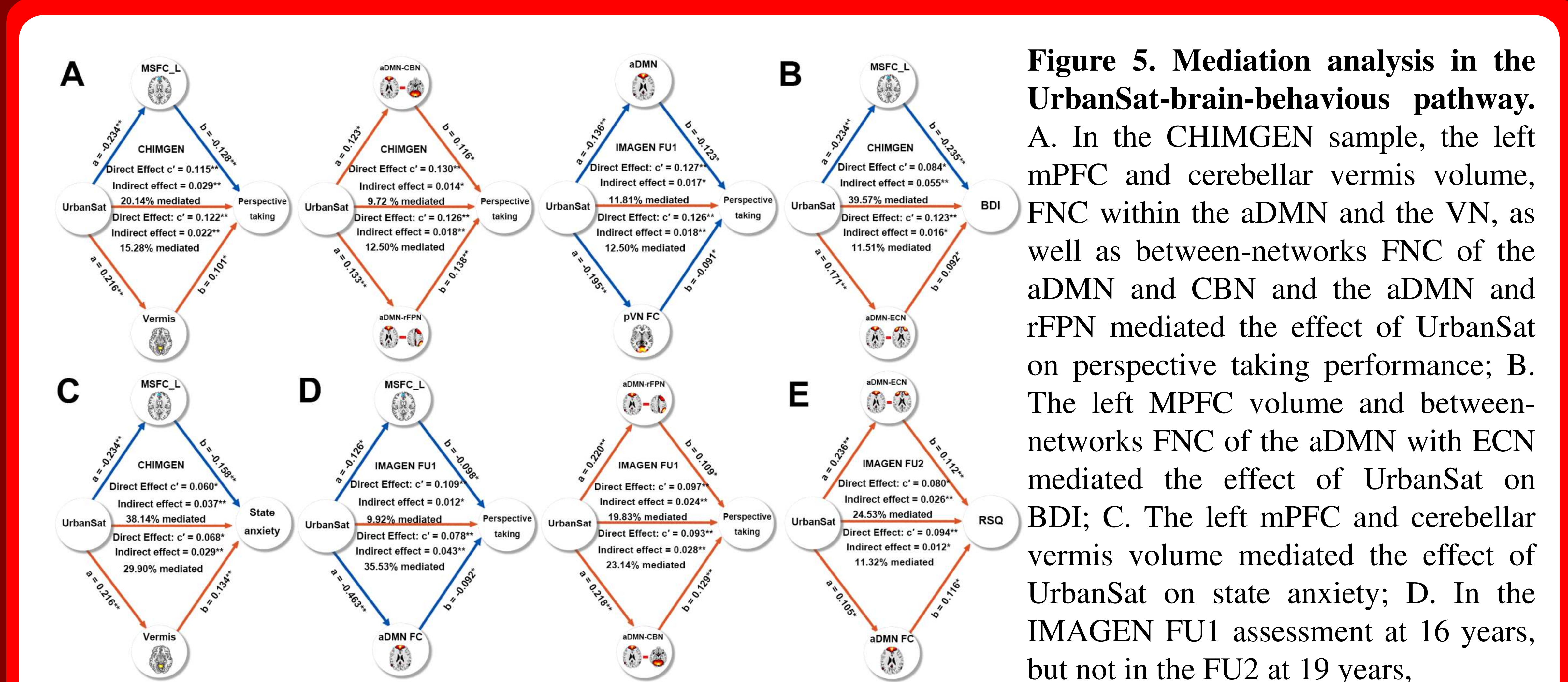

he effect of UrbanSat on perspective taking was mediated by the left MPFC volume, FNC within the aDMN, betweennetworks FNC of the aDMN with CBN and the aDMN with rFPN; E. In the IMAGEN FU2 assessment at 19 years, the effect of UrbanSat on RSQ was mediated by FNC within the aDMN, between-networks FNC of the aDMN with ECN RSQ data were not available at IMAGEN BL sample at 14 years.
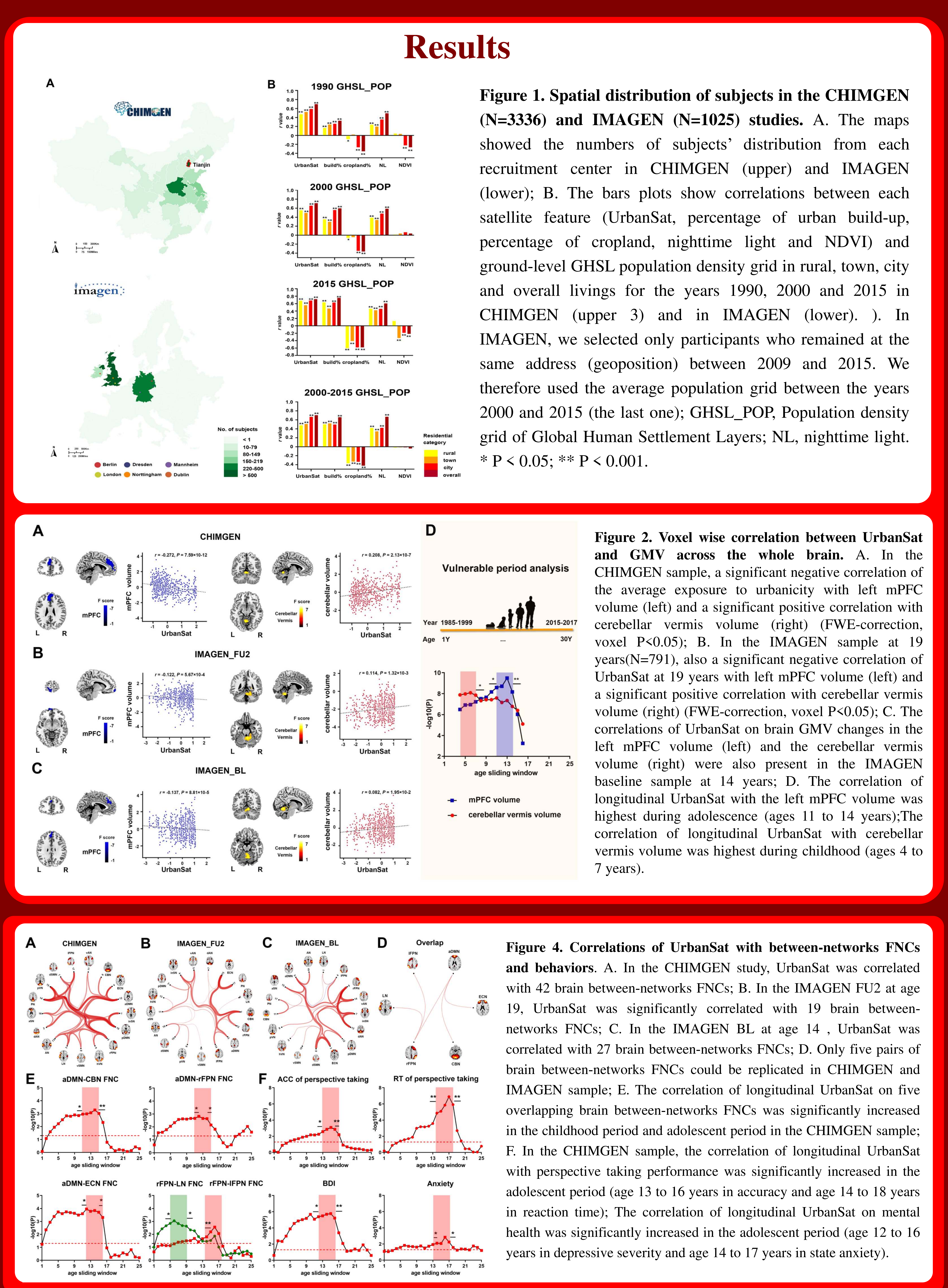

Conclusion

- Using innovative technology, we were able to probe the relationship between urban upbringing with brain change and behaviour in different sociocultural conditions and geographic locations

$\checkmark$ Our findings help to identify shared and distinct determinants of adolescent brain development and mental health in different regions of the world, thus contributing to targeted prevention and early-intervention programs for young people in their unique environment. Our approach may be relevant for public health, policy and urban planning globally.

Nature Medicine, under review 